\title{
Vibrio cholerae Type VI Secretion System Auxiliary Cluster 3 is a Pandemic-associated Mobile Genetic Element
}

\author{
Francis J. Santoriello ${ }^{1}$, Lina Michel ${ }^{1,2}$, Daniel Unterweger ${ }^{3,4}$, Stefan Pukatzki ${ }^{1 *}$ \\ ${ }^{1}$ Department of Immunology and Microbiology, University of Colorado Denver Anschutz Medical \\ Campus, $13001 \mathrm{E} 17$ th PI, Aurora, CO 80045 \\ ${ }^{2}$ Current Address: Institute for Medical Microbiology and Hygiene, University Hospital Ulm, \\ Albert-Einstein-Allee 23, 89081 Ulm, Germany \\ ${ }^{3}$ Max Planck Institute for Evolutionary Biology, August-Thienemann-Straße 2, 24306 Plön, \\ Germany \\ ${ }^{4}$ Institute for Experimental Medicine, Kiel University, Michaelisstraße 5, 24105 Kiel, Germany \\ * Stefan Pukatzki
}

Email: stefan.pukatzki@ucdenver.edu

\section{Keywords}

Vibrio cholerae, Type VI Secretion System, site-specific recombination, mobile DNA, prophage grounding 


\section{Abstract}

All sequenced Vibrio cholerae isolates encode a contact-dependent type VI secretion system (T6SS) in three loci that terminate in a toxic effector and cognate immunity protein (E/I) pair, allowing for competitor killing and clonal expansion in aquatic environments and the host gut. Recent studies have demonstrated variability in the toxic effectors produced by different $V$. cholerae strains and the propensity for effector genes to undergo horizontal gene transfer. Here we demonstrate that a fourth cluster, auxiliary cluster 3 (Aux3), encoding the $\mathrm{E} / \mathrm{l}$ pair $t \mathrm{seH} / \mathrm{tsiH}$, is located directly downstream from two putative recombinases and is flanked by repeat elements resembling att sites. Genomic analysis of $749 \mathrm{~V}$. cholerae isolates, including both pandemic and environmental strains, revealed that Aux3 exists in two states: a $\sim 40 \mathrm{~kb}$ prophage-like element in nine environmental isolates and a $\sim 6 \mathrm{~kb}$ element in pandemic isolates. These findings indicate that Aux3 in pandemic $V$. cholerae is evolutionarily related to an environmental prophage-like element. In both states, Aux3 excises from the chromosome via site-specific recombination to form a circular product, likely priming the module for horizontal transfer. Finally, we show that Aux3 can integrate into the Aux3-naïve chromosome in an integrase-dependent, site-specific manner. This highlights the potential of Aux3 to undergo horizontal transfer by a phage-like mechanism, which based on pandemic coincidence may confer currently unknown fitness advantages to the recipient $V$. cholerae cell.

\section{Significance Statement}

V. cholerae is a human pathogen that causes pandemics affecting 2.8 million people annually (1). The $\mathrm{O} 1 \mathrm{El}$ Tor lineage is responsible for the current pandemic. A subset of non-O1 strains cause cholera-like disease by producing the major virulence factors cholera toxin and toxin co-regulated pilus but fail to cause pandemics. The full set of $V$. cholerae pandemic factors is unknown. Here we describe the type VI secretion system (T6SS) Aux3 element as a largely pandemic-specific factor that is evolutionarily related to an environmental prophage-like element circulating in nonpathogenic strains. These findings shed light on V. cholerae T6SS evolution and indicate the Aux3 element as a pandemic-enriched mobile genetic element. 


\section{Main Text \\ Introduction}

Vibrio cholerae, the causative agent of cholera, is capable of causing natural pandemics. 01 Classical strains caused the first six pandemics, and $\mathrm{O} 1 \mathrm{El}$ Tor strains cause the current $7^{\text {th }}$ pandemic (2-4). Pandemic strains cause diarrheal disease with the major virulence factors cholera toxin (CT) and toxin co-regulated pilus (TCP) (5-8). Several non-O1 strains, however, carry these main virulence factors and cause isolated cases of cholera-like illness without causing pandemic outbreaks (9-11). The full set of factors that make a given $V$. cholerae strain pandemic is unknown.

In its aquatic reservoir and the human small intestine, $V$. cholerae competes with other bacteria and predatory eukaryotic cells. V. cholerae employs the type VI secretion system (T6SS), a contractile nanomachine resembling a T4 bacteriophage tail that kills neighboring competitors through the contact-dependent translocation of toxic effector proteins (12-14). The main components of the T6SS are encoded in three loci (the large cluster, auxiliary cluster 1 (Aux1), and auxiliary cluster 2 (Aux2)), each terminating in an effector/immunity (E/I) pair $(13,15,16)$. While T6 effectors are toxic to distinct bacteria, kin cells are protected by cognate immunity proteins $(16,17)$. It is hypothesized that this allows a strain to clear a niche and propagate clonally (18). Comparative genomic studies of $V$. cholerae T6SS loci have demonstrated that all pandemic strains carry an identical set of effector genes referred to as A-type effectors but environmental strains encode variable effector and immunity subtypes $(19,20)$. Pan-genome hierarchical clustering of $V$. cholerae does not reflect the dispersion of these effector subtypes (20), suggesting horizontal gene transfer (HGT) as a critical player in T6SS E/I evolution. V. cholerae in both the estuarine environment and its human host is exposed to exogenous DNA, bacteriophage, and conjugative elements. Further, chitin-associated $V$. cholerae upregulates the T6SS and natural competence machinery (21-23), driving rapid evolution via inter- and intra-species competition. Recently, horizontal transfer of variable T6SS effectors to chitin-bound V. cholerae was demonstrated in vitro (24). These studies 
indicate the aquatic environment and potentially the host gut as a reservoir for the acquisition of new E/l subtypes.

Some T6SS components are bacteriophage structural homologs (25-27), suggesting that the T6SS is the repurposing of one or more lysogenic phage infections. V. cholerae T6SS loci do not, however, reflect typical prophage genomic organization or encode functional recombinases. Several other virulence factors in V. cholerae such as CT, TCP, and the SXT element are either phage-encoded or acquired by phage-like site-specific recombination (28-32). In each case, integrases and transposases catalyze recombination. These data support the idea that bacteriophage and phage-derived elements play an integral role in the development of pandemic V. cholerae strains.

Recently, Altindis et al. identified a fourth T6SS cluster in V. cholerae. This locus, designated Auxiliary cluster 3 (Aux3), encodes a PAAR adaptor protein, a hydrolase (TseH), and its cognate immunity protein (TsiH) (33). TseH is likely loaded onto the tip of the T6SS with the assistance of the PAAR adaptor. After translocation into the target cell, TseH catalyzes peptidoglycan degradation. Periplasmic TsiH expression neutralizes this degradation. Unlike the three core T6SS loci, Aux3 is not conserved in all sequenced $V$. cholerae strains (34). Here, we demonstrate that Aux3 extends upstream to include an integrase and a transposase, and that phage-like att sites flank the region from the integrase to $t s i H$. By analyzing $749 \mathrm{~V}$. cholerae genomes, we determined that the Aux3 element is found in 572 strains of which 566 encode CT, TCP, and a pandemic Atype T6SS effector set $(19,20)$. Based on phylogenetic analysis of a subset of strains, we show that the Aux3 locus appears to have expanded within the entire pandemic lineage. We further determined that Aux3 is present in nine non-pandemic environmental isolates. The environmental Aux3 locus, however, encodes 42-47 extra bacteriophage homologs and appears to move by HGT, indicating that the pandemic Aux3 locus is likely the evolutionary remnant of a prophage-like element circulating in the aquatic reservoir. Finally, both the environmental and pandemic versions 
of Aux3 excise from the genome by site-specific recombination, but only the environmental Aux3 integrase can catalyze transfer to a naïve strain. These findings highlight this locus as a pandemicassociated mobile genetic element (MGE).

\section{Results}

\section{Phage-like att Sites Flank T6SS Cluster Aux3}

Analysis of the Aux3 locus in O1 El Tor strain N16961 revealed that the genes encoding PAAR, $t s e H$, and $t s i H$ (VCA0284-VCA0286) are immediately downstream from two genes annotated as "phage integrase" (VCA0281, int) and "IS5 transposase" (VCA0282, is5) (Fig. 1A). Sliding-window analysis of the region from VCA0280-VCA0287 reveals blocks of variable GC content within Aux3 compared to the surrounding genomic flanks (Fig. 1A). Based on this proximity to putative recombinases and the differential GC content of this region, we hypothesized that this locus constitutes a potential MGE. Recombinase-encoding MGEs are often flanked by repeat elements (attachment (att) sites) that serve as the locus of enzyme binding and DNA recombination. Alignment of Aux3-encoding and Aux3-naïve V. cholerae strains reveals a single recombinant site on either side of Aux3 indicative of site-specific recombination (SI Appendix, Fig. S1A). We thus probed the intergenic sequences between gcvT and int as well as tsiH and thrS for repetitive sequences and identified two long, direct repeats (referred to as attL1/attL2 and attR1/attR2) separated by approximately 40 bp on either side of the Aux3 locus (Fig. 1B), the second of which contains a stretch of six thymidines resembling the $\lambda$ phage att site (35). Alignment of the att $L 1 /$ att $L 2$

and attR1/attR2 sequences from $\mathrm{O} 1 \mathrm{El}$ Tor strain N16961 with attC1/attC2 from environmental strain DL4215 shows strong homology upstream of attL2/attC2 and downstream of attR2/attC2. These results indicate that the sequence between attL2 and attR2 is Aux3-derived, while the sequence outside these sites is derived from the Aux3-naïve genome. We propose that att2 is the relevant att site for Aux3 recombination (SI Appendix, Fig. S1 B and C). Importantly, attL2 and attR2 are found flanking the Aux3 cluster in all analyzed Aux3-encoding V. cholerae strains, and attC2 exists in a single copy between gcvT and thrS in all Aux3-naïve strains (Fig. 1C). These 
findings demonstrate that the Aux3 cluster extends from VCA0281-VCA0286 and potentially constitutes a MGE capable of excising from the genome by site-specific recombination.

\section{Aux 3 is Conserved and Enriched in 01 Pandemic Strains}

A BLASTN search of the El Tor N16961 Aux3 module in 25 pandemic and environmental $V$. cholerae genomes revealed strong conservation of the Aux3 module in pandemic $\mathrm{O} 1$ strains of both the Classical and El Tor biotypes (SI Appendix, Fig. S1A). All analyzed environmental strains lacked the Aux3 cluster (SI Appendix, Fig. S1A). To determine the scale of Aux3 enrichment in pandemic $V$. cholerae strains, we probed the coincidence of $t s e H$ with the pandemic A-type T6SS effectors tseL, vas $X$, and $\operatorname{vgrG}(19,20)$, as well as $\operatorname{ctx} A B$ and $t c p A$. We performed a Megablast search for these six loci across $749 \mathrm{~V}$. cholerae genomes from the PATRIC database (36) to determine the grade (a weighted score accounting for query coverage as well as pairwise identity) for each locus in each genome. Strains were grouped based on having $>99 \%$ grade to $t s e H$ as well as $>99 \%$ grade to the A-type effectors. Of a total 547 strains with hits for $t s e H, 461$ strains had a grade of $>99 \%$ for $t s e H$, tse L, vas $X$, and $\operatorname{vgrG} 3$, corresponding to an enrichment of $t s e H$ in pandemic strains of $p=2.2 \times 10^{-16}$ by Fisher's Exact Test (SI Appendix, Table S4). Due to the fragmented nature of available $V$. cholerae genomes in the PATRIC database, this enrichment is likely an underestimation. We expanded our analysis to include all strains with $t s e H$ regardless of grade and found that 566 of 572 tse $H$-encoding strains also encoded all five selected pandemic factors (SI Appendix, Fig. S2A). It is important to note that the Aux3 element is absent from nonO1/O139 pathogenic strains that do not cause pandemics but carry the major virulence factors CT and TCP (SI Appendix, Fig. S3). These data demonstrate that Aux3 is enriched in the subset of $V$. cholerae strains with the largest global impact on health.

\section{The Pandemic Aux3 Module Is Evolutionarily Related to a Prophage-like Element}

Our Megablast search for tseH, tseL, vas $X$, and vgrG3 in the $V$. cholerae genomes in the PATRIC database revealed six $t s e H$-encoding strains that lack tse $L$ and $c t x A B$ (SI Appendix, Fig. S2 $A$ and 
B). Three of these strains are environmental O1 strains (2012Env-9, Env390, and 2479-86), two of which encode the toxin co-regulated pilus (2012Env-9 and Env390). The remaining three strains (AM-19226, 1154-74, and P-18748) are nonO1/O139 isolates. Investigation of the region between gcvT and thrS in these strains revealed an Aux3 locus approximately 40kb in length compared to the 6kb-long module found in pandemic strains (Fig. 2A). A Megablast search for this region in NCBI returned three more strains with this elongated Aux3 element (V. cholerae str. 20000, Vibrio sp. 2015V-1076, and Vibrio sp. 2017V-1038). Importantly, attL2 and attR2 flank the Aux3 region in each of these strains (Fig. 1C). Alignment of the Aux3 region in these nine environmental strains reveals variability in the additional sequence between VCA0281 and VCA0283, with most of the variability in the 5' half of the region. Further, all environmental strains lack VCA0282 (SI Appendix, Fig. S4). Analysis of these nine environmental strains by PHASTER (37) predicts that the Aux3 region in non-pandemic strains resembles an intact prophage in both gene content and organization (Fig. 2B and SI Appendix, Fig. S5). These data support the idea that Aux3 exists in

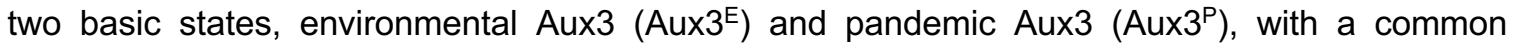
prophage origin. We performed a core genome alignment of 69 pandemic and environmental $V$. cholerae strains as well as 8 Vibrio sp. and one V. mimicus isolate, which shows that the incidence of Aux3 in environmental strains is not reflective of phylogeny (Fig. 3). This scenario leads us to

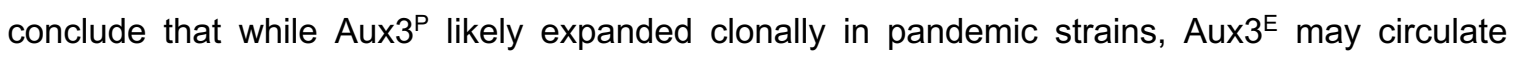
environmentally by HGT. We hypothesize that the evolution of $A u x 3^{P}$ in the pandemic lineage began with the integration of a horizontally transferred phage-like element which then underwent a large deletion event to generate the smaller module (Fig. 2C). We cannot, however, rule out the inverse event, in which Aux $3^{P}$ gained excess prophage-related genes in a large insertion event to form Aux3 ${ }^{\mathrm{E}}$. As all Aux3 ${ }^{\mathrm{E}}$ strains lack is5 (VCA0282) (SI Appendix, Fig. S4), leading us to assume that the insertion of this element occurred in an evolutionary intermediate (Fig. $2 C$ ). We have not yet identified a strain encoding this intermediate Aux $3^{P}$ that lacks the IS5 element. 


\section{Both Aux $^{P}$ and Aux3E Excise to Form a Circular DNA Element by Site-specific Recombination}

A BLASTP search for the Aux3 integrase amino acid sequence returned a conserved domain hit for "integrase P4", a common integrase in temperate phages and pathogenicity islands known to catalyze integration and excision $(30,31,38,39)$. During excision, recombination occurs between attL and attR to reform attC at the chromosomal excision junction and attP on the excised circular DNA element (Fig. 4A) $(40,41)$. Thus, we aimed to determine if Aux3 excises from the genome to form a circular element. We tested this hypothesis by inverted PCR with primers outside of the att sites (P1/P4) and primers inside the att sites facing outward (P2/P3 or P2.2/P3.2) (Fig. 4A). With this design, P1/P4 will only be brought into proximity for amplification upon excision and P2/P3 will only be in the right orientation upon circularization. Two Aux3 ${ }^{E}$ strains (AM-19226 and 1154-74), three Aux3 ${ }^{P}$ strains (N16961, C6706, and A1552), and two Aux3-naïve strains (DL4215 and DL4211) were tested for excision/circularization. After 4 hours of growth, all Aux3 strains excise this element from the genome (Fig. 4B). A band indicative of excision is also evident in the tested environmental strains due to the identical nature of the Aux3-naïve and Aux3-excised states. Further, the circular Aux3 module was present in all Aux3-encoding strains and absent from Aux3naïve strains (Fig. 4B). PCR products were validated by Sanger sequencing against the expected chromosomal and plasmid excision junctions (SI Appendix, Fig. S6A).

To assess the likelihood of Aux3 module transfer to a naïve strain, we measured the incidence of Aux3 excision in each strain by quantitative PCR (qPCR). Primers were designed against the Aux3naïve attC sites to amplify either attC1 or attC2. This experimental setup allows us to quantify excision (reversion to the naïve state) at each site (SI Appendix, Fig. S6B). With two att sites in the intergenic flanks, there are two potential integration states of Aux3. Measuring the reversion to a naïve site at both attC1 and attC2 allows us to determine which attC site is dynamic. Our results show that when normalized to incidence in DL4215 (Aux3-naïve or 100\% excised), attC1 is present at a ratio of approximately 1 in all tested strains (Fig. 4C), indicating that attC1 is constant. The 
incidence of attC2 when normalized to DL4215 is $\sim 1 / 100$ genomes for Aux $3^{E}$ strains and $\sim 1 / 500$ genomes for $\mathrm{Aux}^{\mathrm{P}}$ strains (Fig. $4 \mathrm{C}$ ), supporting attC2 as the site of recombination. It is important to note that primer design was restricted by the proximity of the attC sites (Fig. 1B), leading to an optimal primer efficiency of $\sim 75 \%$. While this is below the desired efficiency, it suggests that the quantification of excision is an underestimation of the true incidence. Time course analysis was performed to assess changes in excision during progression to stationary phase. Strain AM-19226 $\left(\right.$ Aux $\left.3^{\mathrm{E}}\right)$ was sampled at mid $\log (M L), M L+30 \mathrm{~min}, M L+60 \mathrm{~min}, M L+90 \mathrm{~min}$, and $M L+120 \mathrm{~min}$. Quantity of DNA was normalized across time points and taken for PCR. Band intensity increases over the time course for both excision and circularization (Fig. 4D), indicating that excision increases with progression into stationary phase.

\section{Aux $^{\mathrm{E}}$ and $\mathrm{Aux}^{\mathrm{P}}$ Strains Differentially Catalyze Excision and Circularization of Aux3}

To investigate the role of the $\mathrm{Aux} 3^{\mathrm{P}}$-encoded int and is 5 recombinases in modular excision, each recombinase was deleted from the $\mathrm{A} 1552$ chromosome. Aux $3^{\mathrm{P}}$ circularization was assessed by inverted PCR with primers over the circular junction. New circularization primers (P2.2/P3.3) were designed because the original P2 primer binds within the deleted integrase sequence (Fig. 4A). Interestingly, neither single recombinase deletion nor a double knockout abrogated circularization of the Aux3 $3^{P}$ module in $\mathrm{A} 1552$ (Fig. 4E). This could indicate the involvement of an unidentified Aux3-extrinsic recombinase. Conversely, the deletion of the corresponding int gene in the Aux3 strain AM-19226 largely suppressed modular circularization, and trans-complementation of the Aux ${ }^{\mathrm{E}}$ int gene restored circularization to wildtype levels (Fig. 4F).

These data, along with the excision qPCR (Fig. 4C), suggest that there are disparities in the mechanism of site-specific recombination between $A u \times 3^{P}$ and $A u \times 3^{E}$ strains. One potential explanation for this difference is the presence of the IS5 module in Aux3P. A BLASTP search for the int amino acid sequence predicts this protein as a P4-like integrase and tyrosine recombinase. Pairwise alignment of the amino acid sequences of pandemic and environmental int proteins with 
other known tyrosine recombinases shows that both have all appropriate catalytic residues intact and strong homology to each other (SI Appendix, Fig. S7A). At the C-terminus, however, the Aux3 integrase protein is significantly longer than the Aux $3^{P}$ homolog. Closer investigation revealed that the IS5 element in Aux3 ${ }^{\mathrm{P}}$ inserted immediately downstream of the catalytic Y375 residue, blunting the true C-terminal tail of the protein and adding seven nonsense residues encoded by the 5 ' end of the IS5 element (SI Appendix, Fig. S7B). We generated a predictive model of both the full-length and truncated integrase (SI Appendix, Fig. S7 $C$ and $D$ ). While the orientation of the catalytic residues is unaffected, IS5 blunting results in a short, disordered C-terminal tail compared to two tyrosine-rich $\alpha$-helices in the full-length protein (SI Appendix, Fig. S7 C and D). This could explain the decreased incidence of excision seen in Aux $3^{P}$ strains. Counter to this idea, transcomplementation of the $A u x 3^{E}$ integrase into $A 1552 \Delta$ int::Kan does not appear to raise Aux3 excision to environmental levels (Fig. 4F). This suggests that the incidence of excision is not solely reliant on integrase structure, but might be differentially regulated in pandemic strains.

\section{Circular Aux3 Integrates Into an Aux3-naïve Chromosome at attC2 in an int-dependent Manner}

To assess the ability of Aux3 to integrate into the chromosome of an Aux3-naïve V. cholerae strain, we performed conjugative transfer experiments with an Aux3-null V. cholerae recipient strain and a donor E. coli S17 $\lambda$ pir carrying a pKNOCK suicide vector with variable attP sites (SI Appendix,

Fig. S8 $A$ and $B$ ). To generate a recipient strain, we first replaced the Aux3 locus in $V$. cholerae A1552 with a naïve attC site from environmental strain DL4211 (A1552 $\triangle$ Aux3). Next, we

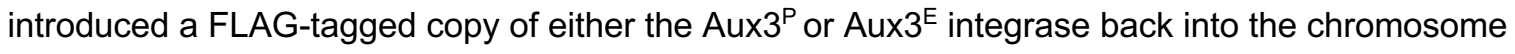
under the control of the $\mathrm{P}_{\mathrm{BAD}}$ promoter on the mini $\mathrm{Tn} 7$ transposon $\left(\mathrm{Tn}:\right.$ :int $^{P}$ or $\mathrm{Tn}::$ int $^{E}$ ), allowing us to induce integrase expression with the addition of arabinose to the culture media. Integrase expression was confirmed in these strains by western blot (SI Appendix, Fig. S8 $C$ and $D)$. It is 
important to note that the $A u \times 3^{\mathrm{P}}$ integrase construct is expressed at much lower levels than the Aux3 $^{\mathrm{E}}$ integrase despite robust expression from the parental plasmid in E. coli (SI Appendix, Fig. S8D). The $P_{B A D}$ promoter region and FLAG-int $P$ construct were verified by Sanger sequencing, and the reduced expression phenomenon was observed in 7 individual clones (data not shown). It is possible that the truncated int $t^{P}$ is being targeted for degradation. Aux3 donor constructs were generated in pKNOCK-Kan to either carry a stretch of circular Aux3 with both attP1 and attP2 intact (pKNOCK-attP $\left.P^{W T}\right)$ or a deletion of the attP2 site (pKNOCK-attP $\left.{ }^{\mathrm{KO}}\right)(\mathrm{SI}$ Appendix, Fig. S8B). This experimental design allowed us to determine which integrase can catalyze integration of the Aux3 element into the naïve chromosome and if the recombination happens in a site-specific manner.

After $24 \mathrm{hr}$ co-culture of donor and recipient under inducing or repressing conditions, mixtures were plated on LB agar with kanamycin (donors), rifampicin/gentamycin (recipients), and rifampicin/gentamycin/kanamycin (transconjugants) to determine respective colony forming units (CFUs) and conjugation frequencies. For all conditions, no significant difference was seen in the recipient or donor counts (Fig. 5A and SI Appendix, Table S5). While all other conditions resulted in conjugation frequencies at or slightly above the limit of detection, transfer of pKNOCK-attP $P^{W T}$ to the induced $\mathrm{Tn}::$ int $^{E}$ recipient resulted in a 3-log increase of conjugation frequency over baseline (Fig. $5 B$ and $C$, and SI Appendix, Fig. S8E and Table S5). The locus of integration was confirmed by PCR with P1/P4 (Fig. $5 D$ ), as an integrated pKNOCK-attP ${ }^{W T}$ results in a 3 kb fragment compared to the 220 bp Aux3-naïve fragment. These results demonstrate that the Aux3 ${ }^{\mathrm{E}}$ integrase is capable of catalyzing recombination between attP2 on circular Aux3 and attC2 on the naïve chromosome, and further indicate that $A u \times 3^{E}$ is a MGE circulating in the aquatic $V$. cholerae reservoir.

\section{Discussion}

Here, we demonstrated that the T6SS Aux3 module is specific mainly to pandemic strains of $V$. cholerae. We further revealed that this locus is the evolutionary remnant of a prophage-like element circulating in the environmental reservoir of non-pathogenic $V$. cholerae strains. The Aux3 ${ }^{\mathrm{E}}$ element 
uses its encoded phage integrase to catalyze site-specific recombination at the flanking att2 sites, forming a circular Aux3 element that is likely primed for horizontal gene transfer to an Aux3-naïve strain of $V$. cholerae. We showed that this locus is partially conserved and expanded within the pandemic lineage of $V$. cholerae. Despite the lack of the majority of its prophage structural and regulatory genes in the pandemic $\mathrm{Aux} 3^{\mathrm{P}}$, this locus maintains its $\mathrm{P} 4$-like integrase and flanking att sites. Site-specific recombination of this locus is conserved at lower levels in pandemic strains, although the Aux 3 integrase does not seem to be necessary for this process. Finally, we show that the $A u x 3^{E}$ integrase is capable of integrating a circular Aux3 element into an Aux3-naïve chromosome at the attC2 site.

The T6SS is a vital defense mechanism for $V$. cholerae and several other pathogenic Gramnegative species in both the host pathogenesis process and interbacterial competition. It is hypothesized that the T6SS is an evolutionary repurposing of a bacteriophage infection $(26,27)$, but the system is conserved so far back in the Vibrio lineage that we have not seen evidence of the initial prophage infections that evolved into the system as it exists today. We believe that our findings offer a snapshot of early T6SS evolution, in which a lysogenic phage infection was degraded to solely the components necessary to increase host fitness. Our results indicate that the pandemic Aux3 locus in V. cholerae is related to an environmentally circulating phage-like element that possibly degraded to form the six-gene pandemic-specific module. The route of transfer in the environmental reservoir is currently unknown, as we have no experimental data to support Aux3 producing its own phage particle. Two potential mechanisms by which Aux $3^{\mathrm{E}}$ could be transferred between strains without making its own phage particle are generalized transduction by environmental lytic phages or chitin-induced natural competence. For the latter mechanism, excision and circularization would likely confer no advantage, as the $V$. cholerae natural competence machinery can foster the transfer of linear genome fragments significantly larger than the Aux $3^{\mathrm{E}}$ module (42). Several V. cholerae modules capable of site specific-recombination, 
however, are transferred by lytic phage transduction (43-45), and it is likely that the circular intermediate is more readily packaged into the transducing phage particle.

In $V$. cholerae, a major role for the T6SS is intraspecies competition and intra-host survival, and the acquisition of new effector proteins could be a key factor in a strains' success or failure in these processes. The phenomenon of T6 effector exchange in V. cholerae has been highlighted $(19,20$, 24), but the mechanism has remained elusive. Here we describe a site-specific recombination mechanism of T6SS effector acquisition for the Aux3 locus. The acquisition of genomic islands by this mechanism is not uncommon in V. cholerae (29-32). For instance, the GIVchS12 element encodes its own integrase, excises from the chromosome to form a circular element, and carries a cluster of T6SS genes including an hcp gene and an E/l pair (32). Aux3, however, can be differentiated from GIVchS12 by its distribution. Like Aux3 ${ }^{\mathrm{E}}$, GIVchS12 circulates in the environmental reservoir of $V$. cholerae by apparent HGT (20), but Aux3 expands into the pandemic lineage. This indicates that the acquisition of $A u \times 3^{E}$ and the eventual reduction to Aux $3^{P}$ may have been an important step in the transition from environmental to pandemic organism.

Further supporting the potential fitness advantage of Aux3, our results show a disparity in the quantity of excision between $A u x 3^{E}$ and Aux $3^{P}$. Truncation of int ${ }^{E}$ appears to have occurred by insertional sequence (IS5 element) interruption to form int $P$. IS5 elements have been shown to drive rapid adaptation in response to environmental stress through either transcriptional regulation of nearby genes or through insertional inactivation (46-49). Here we show that the IS5-truncated int $P$ is expressed at much lower levels than the full-length int ${ }^{E}$, despite having the same promoter and induction conditions. We speculate that truncation of the int gene by IS5 leads to degradation of the Int protein and reduced excision or integration of Aux3. Whether this degradation occurs nonspecifically due to truncation and improper folding or as a specific consequence of the short Cterminal tail added by the IS5 element remains to be shown. Excision also appears to be differentially regulated in pandemic strains. Transcomplementation of int ${ }^{E}$ into pandemic $V$. 
cholerae did not increase excision to Aux $3^{E}$ levels. Over-expression of $i n t^{E}$ in pandemic $V$. cholerae did, however, catalyze increased integration of our Aux3 surrogate vector, while int ${ }^{P}$ overexpression did not. Regulation of Aux3 recombination in pandemic strains may have been re-wired to favor the integration of Aux3 over excision. Genes encoded by the Aux3 element may have conferred a competitive edge to a common ancestor of the pandemic clade, and this advantage was locked into the chromosome by IS5 insertion and shifts in overall regulation of excision. This biological phenomenon is referred to as "phage grounding", and is highly advantageous to the host cell harboring the newly inactivated lysogen (50). This is, in fact, one potential route by which the T6SS itself was first acquired. In the case of Aux3, this advantage would likely be conferred by encoding an extra T6SS effector set, but the role of $t s e H$ in T6SS-dependent competition is still unclear (33). We believe that our findings yield several indications that Aux3 integration was selected for during the evolution of pandemic $V$. cholerae and that further mechanistic studies of the role of $t s e H$ are warranted.

\section{Materials and Methods}

SI Appendix, Supplementary Materials and Methods describes in detail the procedures used in this study, including the following: bacterial growth conditions, mutant strain construction, identification of bacteriophage elements, sequence alignment, Aux3 enrichment analysis, phylogeny and tree building, excision PCR and qPCR, and Aux3 module transfer experiments.

\section{Acknowledgments}

We acknowledge Michelle Dziejman, David Rozak, and Melanie Blokesch for strains and plasmid constructs necessary for the completion of this study. This work was supported by the National Institutes of Health (1R01Al139103-01A1) and the University of Colorado Anschutz Medical Campus. 


\section{References}

1. M. Ali, A. R. Nelson, A. L. Lopez, D. A. Sack, Updated global burden of cholera in endemic countries. PLoS Negl Trop Dis 9, e0003832 (2015).

2. N. Hasan, et al., Genomic diversity of 2010 Haitian cholera outbreak strains. Proc Natl. Acad Sci 109, E2010-E2017 (2012).

3. D. Domman, et al., Integrated view of Vibrio cholerae in the Americas. Science 358, 789-793 (2017).

4. F.-X. Weill, et al., Genomic history of the seventh pandemic of cholera in Africa. Science 358, 785-789 (2017).

5. D. Cassel, Z. Selinger, Mechanism of adenylate cyclase activation by cholera toxin: inhibition of GTP hydrolysis at the regulatory site. Proc Natl Acad Sci USA 74, 3307-11 (1977).

6. D. Gill, R. Meren, ADP-ribosylation of membrane proteins catalyzed by cholera toxin: basis of the activation of adenylate cyclase. Proc Natl. Acad Sci 75, 3050-3054 (1978).

7. D. Herrington, et al., Toxin, toxin-coregulated pili, and the toxR regulon are essential for Vibrio cholerae pathogenesis in humans. J Exp Med. 168, 1487-1492 (1988).

8. K. Thelin, R. Taylor, Toxin-coregulated pilus, but not mannose-sensitive hemagglutinin, is required for colonization by Vibrio cholerae O1 El Tor biotype and $\mathrm{O} 139$ strains. Infect Immun 64, 2853-6 (1996).

9. K. Bishop-Lilly, et al., Genome Sequencing of 15 Clinical Vibrio Isolates, Including 13 NonO1/Non-O139 Serogroup Strains. Genome Announc 2, e00893-14 (2014).

10. B. Haley, et al., Genomic and Phenotypic Characterization of Vibrio cholerae Non-O1 Isolates from a US Gulf Coast Cholera Outbreak. Plos One 9, e86264 (2014).

11. E. Bernardy, M. Turnsek, S. Wilson, C. Tarr, B. Hammer, Diversity of Clinical and Environmental Isolates of Vibrio cholerae in Natural Transformation and Contact-Dependent Bacterial Killing Indicative of Type VI Secretion System Activity. Appl Env. Microb 82, 28332842 (2016).

12. S. Pukatzki, et al., Identification of a conserved bacterial protein secretion system in Vibrio cholerae using the Dictyostelium host model system. P Natl Acad Sci Usa 103, 1528-1533 (2006).

13. S. Pukatzki, M. Steven, S. Miyata, The type VI secretion system: translocation of effectors and effector-domains. Curr Opin Microbiol 12, 11-17 (2009).

14. D. Maclntyre, S. Miyata, M. Kitaoka, S. Pukatzki, The Vibrio cholerae type VI secretion system displays antimicrobial properties. Proc. ... 107, 19520-19524 (2010).

15. J. Zheng, B. Ho, J. Mekalanos, Genetic Analysis of Anti-Amoebae and Anti-Bacterial Activities of the Type VI Secretion System in Vibrio cholerae. Plos One 6, e23876 (2011).

16. T. Dong, B. Ho, Y.-H. Deborah, J. Mekalanos, Identification of T6SS-dependent effector and immunity proteins by Tn-seq in Vibrio cholerae. Proc Natl. Acad Sci 110, 2623-2628 (2013).

17. S. Miyata, D. Unterweger, S. Rudko, S. Pukatzki, Dual Expression Profile of Type VI Secretion System Immunity Genes Protects Pandemic Vibrio cholerae. Plos Pathog $\mathbf{9}$, e1003752 (2013).

18. L. McNally, et al., Killing by Type VI secretion drives genetic phase separation and correlates with increased cooperation. Nat Commun 8, 14371 (2017).

19. D. Unterweger, et al., The Vibrio cholerae type VI secretion system employs diverse effector modules for intraspecific competition. Nat Commun 5, 3549 (2014).

20. P. C. Kirchberger, D. Unterweger, D. Provenzano, S. Pukatzki, Y. Boucher, Sequential displacement of Type VI Secretion System effector genes leads to evolution of diverse immunity gene arrays in Vibrio cholerae. Sci Rep 7, 45133 (2017).

21. K. Meibom, Chitin Induces Natural Competence in Vibrio cholerae. 310, 1824-1827 (2005).

22. M. Scrudato, M. Blokesch, The Regulatory Network of Natural Competence and Transformation of Vibrio cholerae. Plos Genet 8, e1002778 (2012).

23. S. Borgeaud, L. Metzger, T. Scrignari, M. Blokesch, The type VI secretion system of Vibrio cholerae fosters horizontal gene transfer. 347, 63-67 (2015). 
24. J. Thomas, S. Watve, W. Ratcliff, B. Hammer, Horizontal Gene Transfer of Functional Type VI Killing Genes by Natural Transformation. Mbio 8, e00654-17 (2017).

25. S. Pukatzki, A. Ma, A. Revel, D. Sturtevant, J. Mekalanos, Type VI secretion system translocates a phage tail spike-like protein into target cells where it cross-links actin. Proc Natl. Acad Sci 104, 15508-15513 (2007).

26. P. Leiman, et al., Type VI secretion apparatus and phage tail-associated protein complexes share a common evolutionary origin. Proc Natl. Acad Sci 106, 4154-4159 (2009).

27. L. Pell, V. Kanelis, L. Donaldson, P. Howell, A. Davidson, The phage $\lambda$ major tail protein structure reveals a common evolution for long-tailed phages and the type VI bacterial secretion system. Proc Natl. Acad Sci 106, 4160-4165 (2009).

28. M. Waldor, J. Mekalanos, Lysogenic conversion by a filamentous phage encoding cholera toxin. Science 272, 1910-4 (1996).

29. B. Hochhut, M. K. Waldor, Site-specific integration of the conjugal Vibrio cholerae SXT element into prfC. 32, 99-110 (1999).

30. C. Rajanna, et al., The vibrio pathogenicity island of epidemic Vibrio cholerae forms precise extrachromosomal circular excision products. J Bacteriol 185, 6893-901 (2003).

31. R. Murphy, E. Boyd, Three Pathogenicity Islands of Vibrio cholerae Can Excise from the Chromosome and Form Circular Intermediates. J Bacteriol 190, 636-647 (2008).

32. M. Labbate, et al., A genomic island in Vibrio cholerae with VPI-1 site-specific recombination characteristics contains CRISPR-Cas and type VI secretion modules. Sci Rep. 6, 36891 (2016).

33. E. Altindis, T. Dong, C. Catalano, J. Mekalanos, Secretome Analysis of Vibrio cholerae Type VI Secretion System Reveals a New Effector-Immunity Pair. Mbio 6, e00075-15 (2015).

34. C. Crisan, et al., Analysis of Vibrio cholerae genomes identifies new type VI secretion system gene clusters. Genome Biol 20, 163 (2019).

35. A. Landy, W. Ross, Viral integration and excision: structure of the lambda att sites. Science 197, 1147-60 (1977).

36. A. Wattam, et al., Improvements to PATRIC, the all-bacterial Bioinformatics Database and Analysis Resource Center. Nucleic Acids Res 45, D535-D542 (2017).

37. D. Arndt, et al., PHASTER: a better, faster version of the PHAST phage search tool. Nucleic Acids Res 44, W16-W21 (2016).

38. L. S. Pierson, M. L. Kahn, Integration of satellite bacteriophage P4 in Escherichia coli DNA sequences of the phage and host regions involved in site-specific recombination. $J$ Mol Biol 196, 487-496 (1987).

39. D. Esposito, J. Scocca, The integrase family of tyrosine recombinases: evolution of a conserved active site domain. Nucleic Acids Res 25, 3605-3614 (1997).

40. S. Kim, A. Landy, Lambda Int protein bridges between higher order complexes at two distant chromosomal loci attL and attR. Science 256, 198-203 (1992).

41. A. Campbell, Chromosomal insertion sites for phages and plasmids. $J$ Bacteriol 174, 74957499 (1992).

42. N. Matthey, et al., Neighbor predation linked to natural competence fosters the transfer of large genomic regions in Vibrio cholerae. Elife 8, e48212 (2019).

43. E. Boyd, M. Waldor, Alternative mechanism of cholera toxin acquisition by Vibrio cholerae: generalized transduction of CTXPhi by bacteriophage CP-T1. Infect Immun 67, 5898-905 (1999).

44. Y. A. O'Shea, E. Boyd, Mobilization of the Vibrio pathogenicity island between Vibrio cholerae isolates mediated by CP-T1 generalized transduction. FEMS Microbiol Lett 214, 153-7 (2002).

45. S. Udden, et al., Acquisition of classical CTX prophage from Vibrio cholerae 0141 by El Tor strains aided by lytic phages and chitin-induced competence. 105, 11951-11956 (2008).

46. K. Schnetz, B. Rak, IS5: a mobile enhancer of transcription in Escherichia coli. Proc Natl. Acad Sci 89, 1244-1248 (1992).

47. R. G. Sawers, Expression of fnr Is Constrained by an Upstream IS5 Insertion in Certain Escherichia coli K-12 Strains. J. Bacteriol. 187, 2609-2617 (2005). 
bioRxiv preprint doi: https://doi.org/10.1101/868539; this version posted December 8, 2019. The copyright holder for this preprint (which was not certified by peer review) is the author/funder, who has granted bioRxiv a license to display the preprint in perpetuity. It is made available under aCC-BY-NC-ND 4.0 International license.

48. Z. Zhang, M. Saier, A Novel Mechanism of Transposon-Mediated Gene Activation. Plos Genet 5, e1000689 (2009).

49. W. Liang, et al., Sequence polymorphisms of rfbT among the Vibrio cholerae $\mathrm{O} 1$ strains in the Ogawa and Inaba serotype shifts. Bmc Microbiol 13, 1-10 (2013).

50. B. Ramisetty, P. Sudhakari, Bacterial 'Grounded' Prophages: Hotspots for Genetic Renovation and Innovation. Front. Genet. 10, 65 (2019). 
Figures and Tables

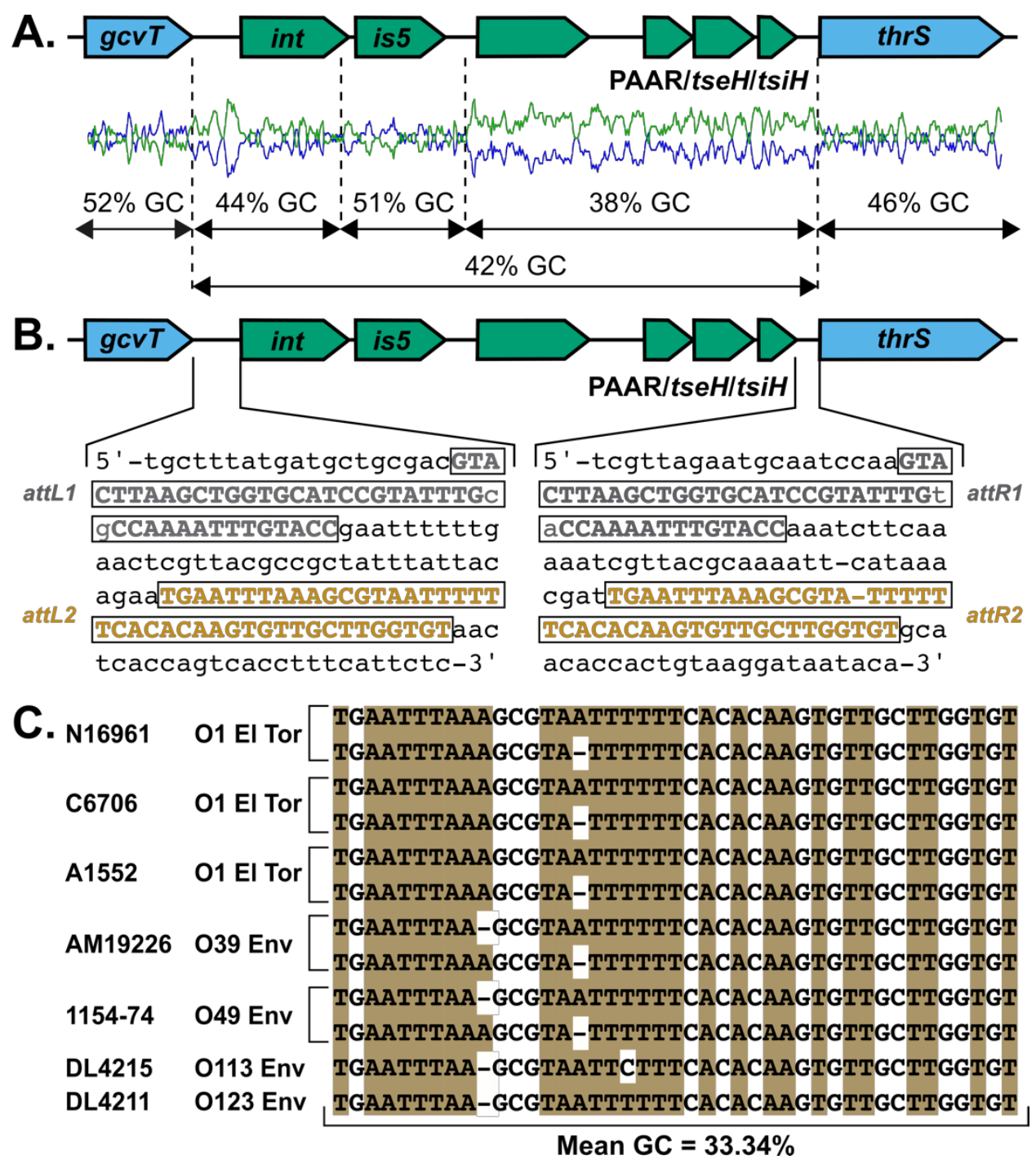

Figure 1. T6SS Aux3 module is flanked by conserved phage-like att sites. $(A)$ Local GC content was analyzed for the N16961 Aux3 cluster and the flanking regions. Aux3 genes are shown in green and the genomic flanks in blue. GC content (blue line) and AT content (green line) were generated using a sliding window of $40 \mathrm{bp}$. $(B)$ Intergenic regions flanking Aux3 were analyzed for repeated sequences. Direct repeat sequences are boxed and shown in grey (attL1/attR1) and orange (attL2/attR2). (C) Alignment of phage-like att site att2 from Aux3-encoding strains (N16961, C6706, A1552, AM-19226, 1154-74) and Aux3-naïve environmental strains (DL4215, DL4211). Both attL2 (top) and attR2 (bottom) are represented for Aux3-encoding strains, and attC2 is shown for each naïve strain. The average GC content is shown, as att sites are typically AT-rich regions. 


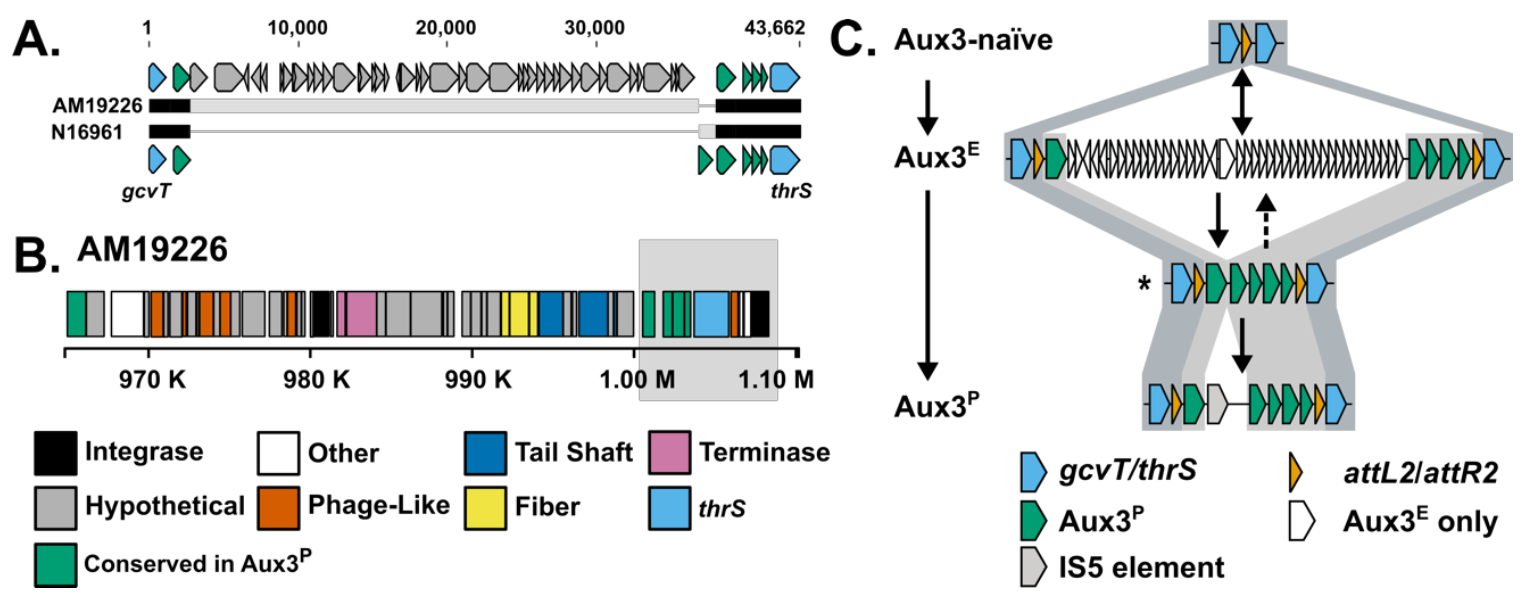

Figure 2. Environmental tse $H$-encoding $V$. cholerae strains carry an alternative prophage-like Aux3 element. (A) MAUVE alignment of the Aux3 locus from pandemic strain N16961 and environmental strain AM-19226. Flanking genes are shown in blue. Pandemic Aux3 genes are shown in green. Black bars indicate nucleotide agreements and grey bars indicate differences. (B) PHASTER genome diagram showing predicted Aux3 prophage region from the leading integrase (VCA0281) through the superintegron integrase (VCA0291) in AM-19226. Coding regions are colored according to homology to broad categories of known phage genes. Light grey box indicates region not called by PHASTER, but verified manually. (C) Schematic of proposed Aux3 module evolution from Aux3-naïve environmental to $A u \times 3^{\mathrm{P}}$ strains. Conserved regions between steps are highlighted in light blue (environmental to pandemic Aux $3^{P}$ ) or grey $\left(A u \times 3^{E}\right.$ to $\left.A u \times 3^{P}\right)$. ${ }^{*}$ indicates a putative unseen intermediate stage in Aux3 evolution. Dashed arrow indicates alternate hypothesis of a large insertion to form $A u \times 3^{E}$. 


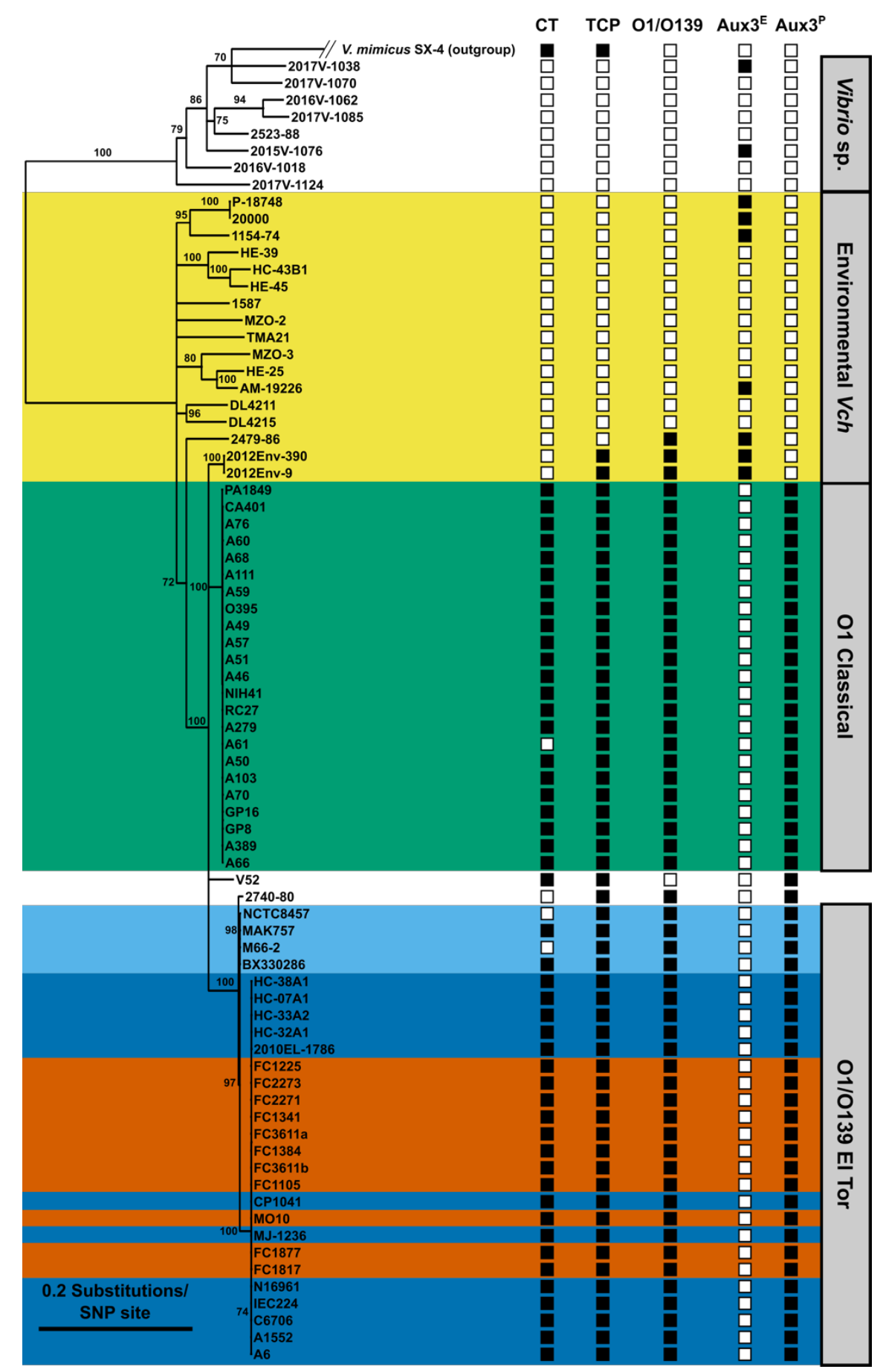

Figure 3. The Aux3 element is enriched in pandemic $V$. cholerae and sporadically distributed in environmental strains. A phylogenetic tree was constructed using the GTR + Gamma Maximum likelihood model in RAxML based on core genome SNP alignment of 69 V. cholerae, 8 Vibrio sp., and $1 \mathrm{~V}$. mimicus genome sequences. Bootstrapping support values are indicated next to their respective branches. Nodes with support values $<70$ were collapsed. Presence (black square) or absence (white square) of CT, TCP, O1/O139 antigen, and the Aux3 $3^{\mathrm{E}}$ or Aux3 $3^{\mathrm{P}}$ module is indicated. Environmental (yellow), O1 Classical (green), Pre-7th Pandemic O1 El Tor (light blue), 7th Pandemic $\mathrm{O} 1 \mathrm{El}$ Tor (dark blue), and $\mathrm{O} 139$ (red) strains are highlighted. 


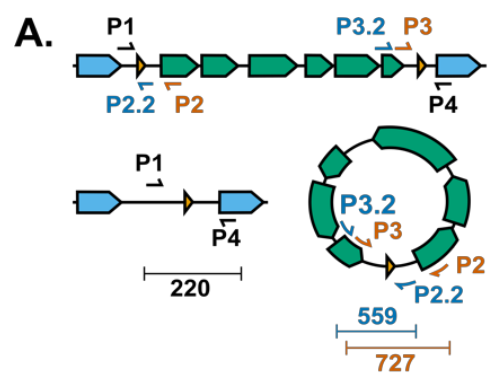

B.
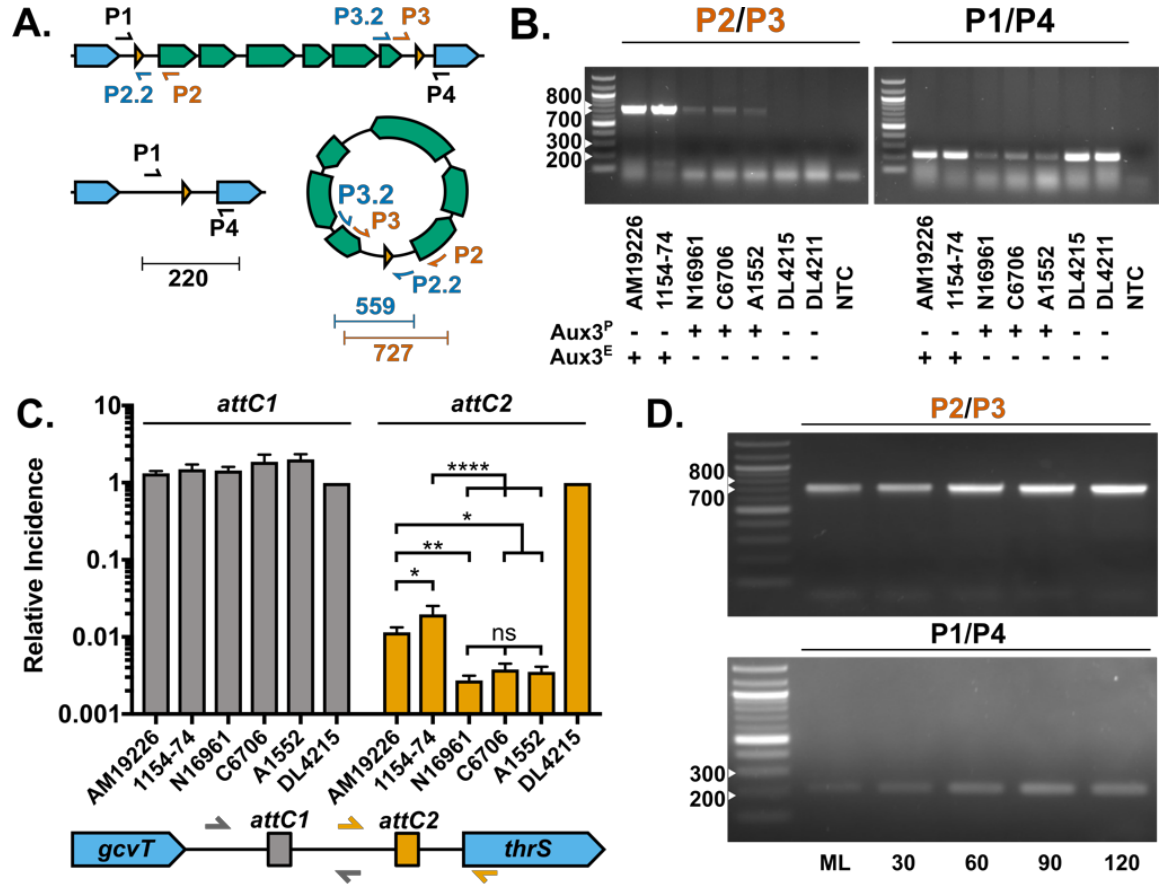

E.

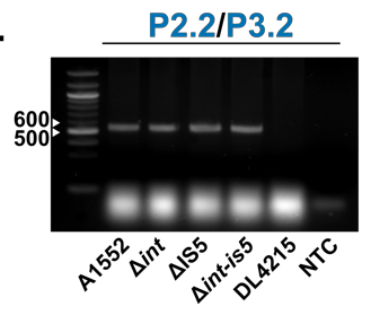

F.

P2.2/P3.2

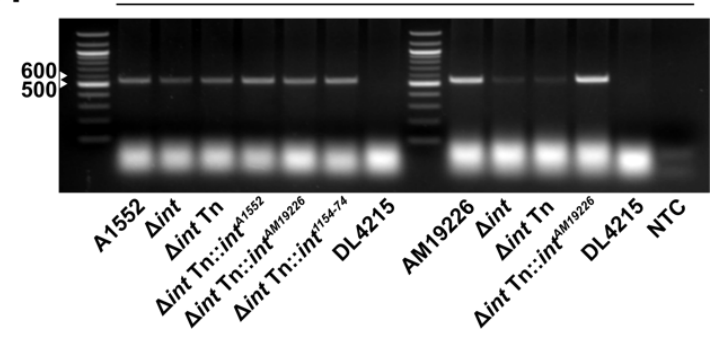

Figure 4. Both $A u \times 3^{E}$ and $A u x 3^{P}$ modules excise from the chromosome to form a circular intermediate. $(A)$ Inverse PCR schematic showing integrated and excised Aux $3^{\mathrm{P}}$. Aux3 genes are green, genomic flanks are blue, and att sites are orange triangles. Primers are represented by arrows and expected band sizes are shown. (B) PCR amplification of excision junctions, attP (P2/P3) and attC (P1/P4), on Aux3E (AM-19226, 1154-74), Aux3 ${ }^{\mathrm{P}}$ (N16961, C6706, A1552), and Aux3-naïve (DL4215, DL4211) strains. (C) Quantification of Aux3 excision by qPCR with primers designed against the naïve att sites attC1 and attC2 (SI Appendix, Fig. S6B) on gDNA from Aux3 ${ }^{\mathrm{E}}$ and $A u \times 3^{P}$ strains. Incidence of excision levels were determined by absolute quantification and normalization to DL4215. Error bars indicate \pm SD. Significance was determined by a one-way ANOVA with Tukey's multiple comparisons test (ns $=$ non-significant, ${ }^{*}=p<0.05,{ }^{* *}=p<0.01$, $* * * *=p<0.0001)$. (D) PCR amplification of excision junctions, attP (P2/P3) and attC (P1/P4) on AM-19226 (Aux3 $3^{E}$ ) DNA over a time course from mid log (ML) to ML + 120 min. Samples were normalized to $10 \mathrm{ng}$ at each time point. (E) Circular (P2.2/P3.2) excision junction PCR from crude DNA extracts of $\mathrm{A} 1552\left(\mathrm{Aux} 3^{\mathrm{P}}\right.$ ) wildtype, $\mathrm{A} 1552$ single and double recombinase null mutants, and DL4215. (F) Circular (P2.2/P3.2) excision junction PCR from crude DNA extracts of A1552 (Aux3 ${ }^{\mathrm{P}}$ ) and AM-19226 (Aux $3^{\mathrm{E}}$ ) wildtype strains, associated int-null mutants, and DL4215. Null mutants from each strain were trans-complemented with an empty $\mathrm{mTn} 7(\mathrm{Tn}), \mathrm{mTn} 7$ with the native integrase, or mTn7 with the opposing Aux3-type integrase ("integrase swap"). For all gel images, white arrows indicate ladder band sizes. 
A.

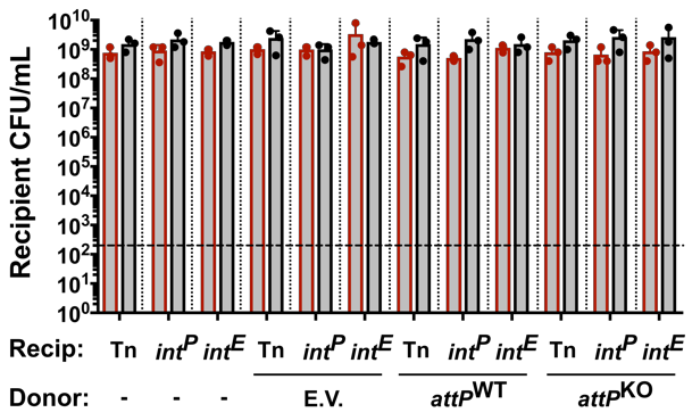

C.

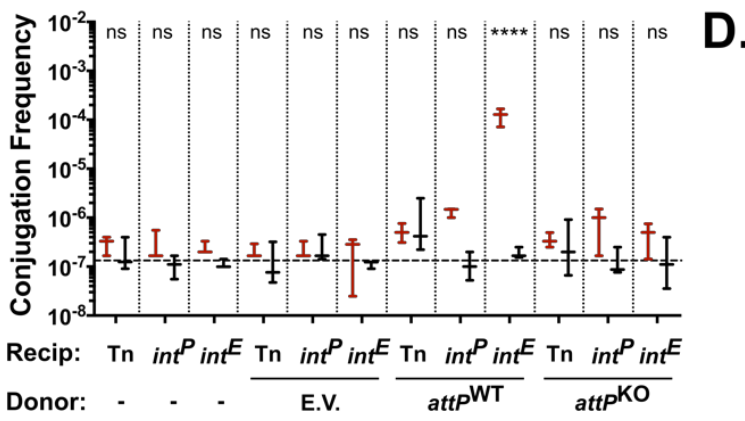

$0.1 \%$ Arabinose
B.

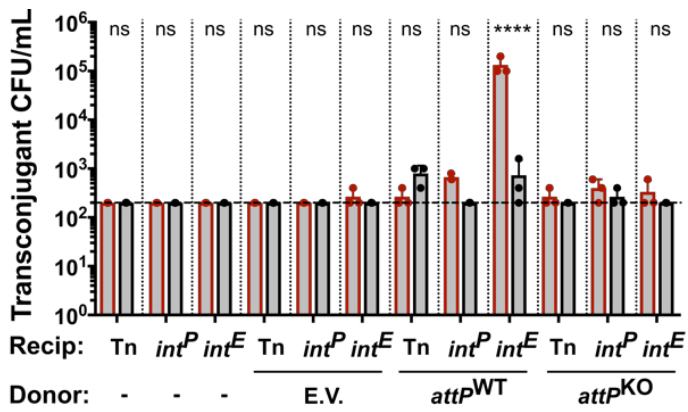

D.

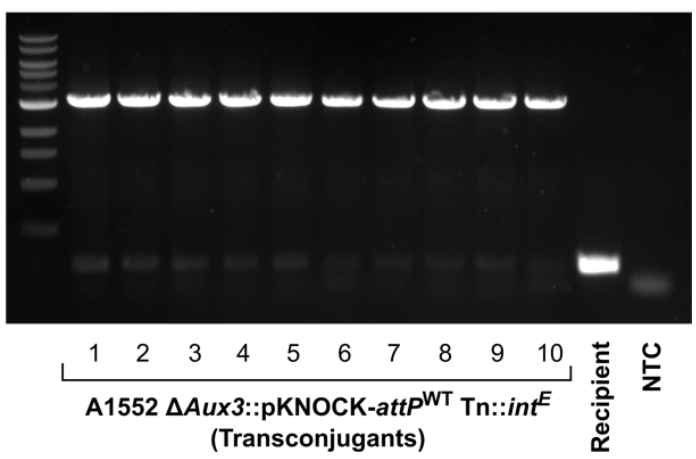

Figure 5. The $A u \times 3^{E}$ integrase catalyzes integration of circular Aux3 into the naïve attC site. $(A)$ Quantification of viable counts of total recipient $V$. cholerae cells $\left(\mathrm{Rif}^{R} / \mathrm{Gent}^{R}\right)$ from conjugative transfer experiments. $(B)$ Quantification of viable counts of transconjugant $V$. cholerae cells $\left(\mathrm{Rif}^{\mathrm{R}} / \mathrm{Gent}^{\mathrm{R}} / \mathrm{Kan}^{\mathrm{R}}\right)$ from conjugative transfer experiments. (C) Conjugation frequency from transfer experiments as determined by transconjugant counts divided by total recipient counts. $(D)$ PCR verification of pKNOCK-attP ${ }^{\mathrm{WT}}$ integration at the defined Aux3 locus with primers P1 and P4 (Fig. $4 A)$. For all quantitative experiments arabinose-induced experiments are shown in red and dextrose control experiments are shown in black. Horizontal dashed line indicates the limit of detection. All quantitative experiments were performed in triplicate. Horizontal bars represent the mean and error bars indicate \pm SD. Significance was determined by a 2 way ANOVA with Sidak's multiple comparisons test (ns = non-significant, $\left.{ }^{* * * *}=p<0.0001\right)$. E.V. $=$ S17 $\lambda$ pir;pKNOCK-Kan; attPWT $=$

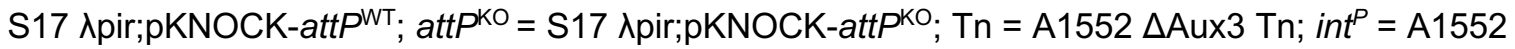
$\Delta$ Aux3 Tn::int ${ }^{P} ;$ int $^{E}=\mathrm{A} 1552 \Delta$ Aux3 Tn::int ${ }^{E}$. 